\title{
Unbounded Solutions of a Boundary Value Problem for Abstract $n$ th-Order Differential Equations on an Infinite Interval
}

\author{
Zhenbin Liu, ${ }^{1,2}$ Lishan Liu, ${ }^{2,3}$ Yonghong $\mathrm{Wu}^{3}$ and Jing $\mathrm{Zhao}^{1}$ \\ ${ }^{1}$ School of Sciences, Qingdao Agricultural University, Shandong 266109, Qingdao, China \\ ${ }^{2}$ School of Mathematical Sciences, Qufu Normal University, Shandong 273165, Qufu, China \\ ${ }^{3}$ Department of Mathematics and Statistics, Curtin University of Technology, Perth 6845, WA, Australia
}

Correspondence should be addressed to Lishan Liu, lls@mail.qfnu.edu.cn

Received 29 January 2007; Revised 28 May 2007; Accepted 17 October 2007

Recommended by Aizicovici Sergiu

The existence of unbounded nonnegative solutions of a boundary value problem for $n$ th-order differential equations defined on an infinite interval is obtained by means of the Mönch fixed-point theorem. An example is then presented to demonstrate the application of our results.

Copyright ( 92008 Zhenbin Liu et al. This is an open access article distributed under the Creative Commons Attribution License, which permits unrestricted use, distribution, and reproduction in any medium, provided the original work is properly cited.

\section{Introduction}

Over the last few years, many researchers have focused their research on the study of boundary value problems for nonlinear differential and integral equations defined on an infinite interval, and various theoretical results have been obtained [1-8]. In [4], the existence of multiple positive solutions of a boundary value problem (BVP) for $n$ th-order nonlinear impulsive integral-differential equations defined on an infinite interval in a Banach space is obtained by means of the fixed-point index theory of completely continuous operators. However, the result requires the use of the measures of noncompactness condition $\alpha\left(f\left(t, P_{r}, \ldots, P_{r}\right)\right)=0$ (where $\left.P_{r}=\{x \in P:\|x\| \leq r\}\right)$ and the normal and solid cone $P$ in a real Banach space. In [6], by using the Mönch fixed-point theorem, a class of infinite boundary value problems for first-order impulsive differential equations in a Banach space is considered and the existence of positive solutions is obtained, but the solutions are limited to bounded solutions only.

To generalize and further develop the existing results in this field, in this paper we discuss the existence of unbounded solutions for a class of $n$ th-order nonlinear differential equations defined on an infinite interval in a Banach space by using the Mönch fixed-point theorem under certain conditions weaker than those in [4]. The boundary value problemin question is 
as follows:

$$
\begin{gathered}
u^{(n)}(t)=f\left(t, u(t), u^{\prime}(t), \ldots, u^{(n-1)}(t)\right) \\
u^{(i)}(0)=\beta_{i} u^{i+1}(0), \quad i=0,1, \ldots, n-2 \\
u^{(n-1)}(+\infty)=\beta u^{(n-1)}(0)
\end{gathered}
$$

where $J=[0,+\infty), u^{(n-1)}(+\infty)=\lim _{t \rightarrow+\infty} u^{(n-1)}(t), \beta>1, \beta_{i}>0(i=0,1, \ldots, n-2), f \in C[J \times E \times$ $\cdots \times E, E]$, in which $(E,\|\cdot\|)$ is a real Banach space.

Let $C[J, E]$ be the space of all continuous functions $u: J \rightarrow E$, and let $L[J, E]$ be the Banach space of all strongly measurable functions $u: J \rightarrow E$ with $\int_{J}\|u(t)\| d t<+\infty$, equipped with the norm $\|u\|_{1}=\int_{J}\|u(t)\| d t$. Let $C_{e}[J, E]=\left\{u \in C[J, E]: e^{-t}\|u(t)\| \rightarrow 0\right.$ for $\left.t \rightarrow+\infty\right\}$, then it is clear that $C_{e}[J, E]$ is a Banach space with norm

$$
\|u\|_{e}=\sup _{t \in J}\left\{e^{-t}\|u(t)\|\right\}
$$

Let $D_{e}^{n-1}[J, E]=\left\{u \in C^{n-1}[J, E]: \lim _{t \rightarrow+\infty} e^{-t}\left\|u^{(i)}(t)\right\| \longrightarrow 0, i=0,1, \ldots, n-1\right\}$, then it is also easy to see that $D_{e}^{n-1}[J, E]$ is a Banach space with norm

$$
\|u\|_{D}=\max \left\{\|u\|_{e},\left\|u^{\prime}\right\|_{e}, \ldots,\left\|u^{(n-1)}\right\|_{e}\right\} .
$$

Let $P$ be a cone of the Banach space $E, C_{e}[J, P]=\left\{u \in C_{e}[J, E]: u(t) \geq \theta, t \in J\right\}$, and $D_{e}^{n-1}[J, P]=\left\{u \in D_{e}^{n-1}[J, E]: u^{(i)}(t) \geq \theta, t \in J, i=0,1, \ldots, n-1\right\}$, where $\theta$ denotes the, zero element of $E$. Then, it is obvious that $C_{e}[J, P]$ is a cone in space $C_{e}[J, E]$, and $D_{e}^{n-1}[J, P]$ is a cone in space $D_{e}^{n-1}[J, E]$.

Definition 1.1. A function $u \in D_{e}^{n-1}[J, P] \cap C^{n}[J, E]$ is called a nonnegative solution of BVP (1.1) if $u(t)$ satisfies (1.1) for $t \in J$.

The rest of the paper is organized as follows. In Section 2, we give some lemmas which provide a theoretical basis for the proof of our main results. The main theorem is presented and proved in Section 3. In Section 4, an example is given to demonstrate the application of our results.

\section{Some lemmas}

Here we first list some assumptions to be used throughout the rest of the paper.

$\left(\mathrm{H}_{1}\right)$ There exist $a(t)$ and $b_{i}(t) \in C\left[J, \mathbb{R}_{+}\right]$such that $a(t), e^{t} b_{i}(t) \in L\left[J, \mathbb{R}_{+}\right](i=$ $0,1,2, \ldots, n-1)$ and

$$
\begin{gathered}
\| f\left(t, x_{0}, x_{1}, \ldots, x_{n-1}\left\|\leq a(t)+\sum_{i=0}^{n-1} b_{i}(t)\right\| x_{i} \| \quad \forall t \in J, x_{i} \in E\right. \\
\sum_{i=0}^{n-1} b_{i}^{*}<\frac{\beta-1}{\beta^{*}+\beta^{\prime}} \quad \beta^{*}=\max _{0 \leq j \leq n-2}\left\{\prod_{i=j}^{n-2} \beta_{i}\right\}, \quad b_{i}^{*}=\int_{0}^{+\infty} e^{t} b_{i}(t) d t<+\infty, \quad i=0,1,2, \ldots, n-1 .
\end{gathered}
$$


$\left(\mathrm{H}_{2}\right)$ There exists $l_{i}(t) \in C\left[J, \mathbb{R}_{+}\right]$such that $e^{t} l_{i}(t) \in L\left[J, \mathbb{R}_{+}\right](i=0,1, \ldots, n-1)$,

$$
\begin{gathered}
\alpha\left(f\left(t, D_{0}, D_{1}, \ldots, D_{n-1}\right)\right) \leq \sum_{i=0}^{n-1} l_{i}(t) \alpha\left(D_{i}\right) \quad \forall t \in J, D_{i} \subset E, \\
\frac{2\left(\beta^{*}+\beta\right)}{\beta-1} \sum_{i=0}^{n-1} l_{i}<1, \quad l_{i}=\int_{0}^{+\infty} e^{t} l_{i}(t) d t<+\infty, \quad i=0,1, \ldots, n-1,
\end{gathered}
$$

where $\alpha(\cdot)$ denotes the Kuratowski measure of noncompactness in $E$. For details on the definition and properties of the measure of noncompactness, the reader is referred to $[9,10]$.

In the following, we give various lemmas which are to be used for the proof of the main results to be presented in Section 3.

Lemma 2.1 (see [4]). Let $\left(H_{1}\right)$ be satisfied. Then $u \in D_{e}^{n-1}[J, P] \cap C^{n}[J, E]$ is a solution of $B V P(1.1)$ if and only if $u \in D_{e}^{n-1}[J, P]$ is a solution of the following integral equation:

$$
\begin{aligned}
u(t)= & \frac{1}{\beta-1}\left[\sum_{j=0}^{n-2}\left(\prod_{i=j}^{n-2} \beta_{i}\right) \frac{t^{j}}{j !}+\frac{t^{n-1}}{(n-1) !}\right] \int_{0}^{+\infty} f\left(s, u(s), u^{\prime}(s), \ldots, u^{(n-1)}(s)\right) d s \\
& +\frac{1}{(n-1) !} \int_{0}^{t}(t-s)^{n-1} f\left(s, u(s), u^{\prime}(s), \ldots, u^{(n-1)}(s)\right) d s .
\end{aligned}
$$

Proof. If $u \in D_{e}^{n-1}[J, P] \cap C^{n}[J, E]$ is a solution of BVP (1.1), then by condition $\left(\mathrm{H}_{1}\right)$ we have the convergence of the infinite integral

$$
\int_{0}^{+\infty} f\left(s, u(s), u^{\prime}(s), \ldots, u^{(n-1)}(s)\right) d s
$$

Integrating the first equation in (1.1) from 0 to $t$, we have

$$
u^{(n-1)}(t)-u^{(n-1)}(0)=\int_{0}^{t} f\left(s, u(s), u^{\prime}(s), \ldots, u^{(n-1)}(s)\right) d s
$$

By virtue of $u^{(n-1)}(+\infty)=\beta u^{(n-1)}(0)$, let $t \rightarrow+\infty$ in (2.5), we get

$$
u^{(n-1)}(0)=\frac{1}{\beta-1} \int_{0}^{+\infty} f\left(s, u(s), u^{\prime}(s), \ldots, u^{(n-1)}(s)\right) d s
$$

From the second equation in (1.1), we have

$$
u^{(i)}(0)=\beta_{i} u^{(i+1)}(0)=\beta_{i} \beta_{i+1} u^{(i+2)}(0)=\beta_{i} \beta_{i+1} \beta_{n-2} u^{(n-1)}(0)=\prod_{j=i}^{n-2} \beta_{j} u^{(n-1)}(0) .
$$


Substituting (2.6) into (2.7) yields for $i=0,1, \ldots, n-2$,

$$
u^{(i)}(0)=\frac{1}{\beta-1} \prod_{j=i}^{n-2} \beta_{j} \int_{0}^{+\infty} f\left(s, u(s), u^{\prime}(s), \ldots, u^{(n-1)}(s)\right) d s
$$

From (2.5) and (2.6), we have

$$
u^{(n-1)}(t)=\frac{1}{\beta-1} \int_{0}^{+\infty} f\left(s, u(s), u^{\prime}(s), \ldots, u^{(n-1)}(s)\right) d s+\int_{0}^{t} f\left(s, u(s), u^{\prime}(s), \ldots, u^{(n-1)}(s)\right) d s
$$

Integrating (2.9) from 0 to $t$ and using (2.8) for $i=n-2$, we have

$$
\begin{aligned}
& u^{(n-2)}(t) \\
& =u^{(n-2)}(0)+\frac{t}{\beta-1} \int_{0}^{+\infty} f\left(s, u(s), u^{\prime}(s), \ldots, u^{(n-1)}(s)\right) d s+\int_{0}^{t} d s \int_{0}^{s} f\left(w, u(w), \ldots, u^{(n-1)}(w)\right) d w \\
& =\left(\frac{\beta_{n-2}}{\beta-1}+\frac{t}{\beta-1}\right) \int_{0}^{+\infty} f\left(s, u(s), u^{\prime}(s), \ldots, u^{(n-1)}(s)\right) d s+\int_{0}^{t}(t-s) f\left(s, u(s), u^{\prime}(s), \ldots, u^{(n-1)}(s)\right) d s
\end{aligned}
$$

It is not difficult to show by mathematical induction that $u$ satisfies (2.3). Conversely, if $u \in$ $D_{e}^{n-1}[J, P]$ is a solution of (2.3), then direct differentiation of (2.3) gives

$$
\begin{aligned}
u^{(i)}(t)= & \frac{1}{\beta-1}\left[\sum_{j=i}^{n-2}\left(\prod_{s=j}^{n-2} \beta_{s}\right) \frac{t^{j-i}}{(j-i) !}+\frac{t^{n-i-1}}{(n-i-1) !}\right] \int_{0}^{+\infty} f\left(s, u(s), u^{\prime}(s), \ldots, u^{(n-1)}(s)\right) d s \\
& +\frac{1}{(n-i-1) !} \int_{0}^{t}(t-s)^{n-i-1} f\left(s, u(s), u^{\prime}(s), \ldots, u^{(n-1)}(s)\right) d s, \quad i=0,1, \ldots, n-2, \\
u^{(n-1)}(t) & =\frac{1}{\beta-1} \int_{0}^{+\infty} f\left(s, u(s), u^{\prime}(s), \ldots, u^{(n-1)}(s)\right) d s+\int_{0}^{t} f\left(s, u(s), u^{\prime}(s), \ldots, u^{(n-1)}(s)\right) d s, \\
u^{(n)}(t) & =f\left(t, u(t), u^{\prime}(t), \ldots, u^{(n)}(t)\right) \quad \forall t \in J .
\end{aligned}
$$

Consequently, $u \in C^{n}[J, E]$, and by (2.11) and (2.12), it is easy to see that $u(t)$ satisfies (1.1). The proof of Lemma 2.1 is completed. 
We now consider an operator $A$ defined by

$$
\begin{aligned}
(A u)(t)= & \frac{1}{\beta-1}\left[\sum_{j=0}^{n-2}\left(\prod_{s=j}^{n-2} \beta_{s}\right) \frac{t^{j}}{j !}+\frac{t^{n-1}}{(n-1) !}\right] \int_{0}^{+\infty} f\left(s, u(s), u^{\prime}(s), \ldots, u^{(n-1)}(s)\right) d s \\
& +\frac{1}{(n-1) !} \int_{0}^{t}(t-s)^{n-1} f\left(s, u(s), u^{\prime}(s), \ldots, u^{(n-1)}(s)\right) d s \quad \forall t \in J .
\end{aligned}
$$

By Lemma 2.1, $u \in D_{e}^{n-1}[J, P] \cap C^{n}[J, E]$ is a solution of BVP (1.1) if and only if $u$ is a fixed point of the operator $A$ in $u \in D_{e}^{n-1}[J, P]$.

Lemma 2.2 (see $[10,11,13])$. Let $E$ be a Banach space, let $J_{0}=[a, b]$ be a finite interval, and let $H \subset C\left[J_{0}, E\right]$ be a countable set. Assume that there exists $\rho \in L\left[J_{0}, \mathbb{R}_{+}\right]$such that $\|u(t)\| \leq \rho(t), t \in$ $J_{0}, u \in H$. Then $\alpha(\{u(t): u \in H\}) \in L\left[J_{0}, \mathbb{R}_{+}\right]$and

$$
\alpha\left(\left\{\int_{J_{0}} u(t) d t: u \in H\right\}\right) \leq 2 \int_{J_{0}} \alpha(\{u(t): u \in H\}) d t
$$

Lemma 2.3. Let $E$ be a Banach space, and $H \subset C[J, E]$. If $H$ is a countable set and there exists $\rho \in L\left[J, \mathbb{R}_{+}\right]$such that $\|u(t)\| \leq \rho(t), t \in J, u \in H$, then $\alpha(\{u(t): u \in H\})$ is integrable on $J$, and

$$
\alpha\left(\left\{\int_{0}^{+\infty} u(t) d t: u \in H\right\}\right) \leq 2 \int_{0}^{+\infty} \alpha(\{u(t): u \in H\}) d t
$$

Proof. By $\|u(t)\| \leq \rho(t)$ for any $u \in H$ and $t \in J$, we get $u(t) \in L[J, E]$ for all $u \in H$ and $\alpha(\{u(t): u \in H\}) \leq 2 \rho(t)$. As $\rho(t) \in L\left[J, \mathbb{R}_{+}\right], \alpha(\{u(t): u \in H\})$ is integrable in $J$. For any $\varepsilon>0$, from $\rho(t) \in L\left[J, \mathbb{R}_{+}\right]$, there exists $T_{0}>0$ such that $\int_{t}^{+\infty} \rho(s) d s<\varepsilon$ for any $t>T_{0}$. So for any $t>T_{0}$ and $u \in H$, we have

$$
\left\|\int_{0}^{t} u(s) d s-\int_{0}^{+\infty} u(s) d s\right\|=\left\|\int_{t}^{+\infty} u(s) d s\right\|<\int_{t}^{+\infty} \rho(s) d s<\varepsilon
$$

Let

$$
S(t)=\left\{\int_{0}^{t} u(s) d s: u \in H\right\}, \quad T=\left\{\int_{0}^{+\infty} u(s) d s: u \in H\right\} .
$$

By (2.16) for any $t>T_{0}$ and $u \in H$, we obtain

$$
d\left(\int_{0}^{t} u(s) d s, T\right)=\inf _{u \in H}\left\{\left\|\int_{0}^{t} u(s) d s-\int_{0}^{+\infty} u(s) d s\right\|\right\} \leq\left\|\int_{0}^{t} u(s) d s-\int_{0}^{+\infty} u(s) d s\right\|<\varepsilon,
$$


where $d$ denotes the distance of a point to a set. Using the same method, we have

$$
d\left(\int_{0}^{+\infty} u(s) d s, S(t)\right)<\varepsilon, \quad t>T_{0}, u \in H
$$

So

$$
\sup _{u \in H} d\left(\int_{0}^{t} u(s) d s, T\right) \leq \varepsilon, \quad \sup _{u \in H} d\left(\int_{0}^{+\infty} u(s) d s, S(t)\right) \leq \varepsilon .
$$

Hence, for any $t>T_{0}$, we have

$$
\begin{aligned}
& d_{h}\left(\left\{\int_{0}^{t} u(s) d s: u \in H\right\},\left\{\int_{0}^{+\infty} u(s) d s: u \in H\right\}\right) \\
& \quad=\max \left\{\sup _{u \in H} d\left(\int_{0}^{t} u(s) d s, T\right), \sup _{u \in H} d\left(\int_{0}^{+\infty} u(s) d s, S(t)\right)\right\} \leq \varepsilon,
\end{aligned}
$$

where $d_{h}\left(D_{1}, D_{2}\right)$ denotes the Hausdorff distance of the sets $D_{1}$ and $D_{2}$, that is,

$$
d_{h}\left(D_{1}, D_{2}\right)=\max \left\{\sup _{x \in D_{1}} d\left(x, D_{2}\right), \sup _{x \in D_{2}} d\left(x, D_{1}\right)\right\} .
$$

So, by (2.21), we obtain for any $t>T_{0}$,

$$
\begin{aligned}
& \left|\alpha\left(\left\{\int_{0}^{t} u(s) d s: u \in H\right\}\right)-\alpha\left(\left\{\int_{0}^{+\infty} u(s) d s: u \in H\right\}\right)\right| \\
& \quad \leq 2 d_{h}\left(\left\{\int_{0}^{t} u(s) d s: u \in H\right\},\left\{\int_{0}^{+\infty} u(s) d s: u \in H\right\}\right) \leq 2 \varepsilon
\end{aligned}
$$

Hence, we have

$$
\lim _{t \rightarrow+\infty} \alpha\left(\left\{\int_{0}^{t} u(s) d s: u \in H\right\}\right)=\alpha\left(\left\{\int_{0}^{+\infty} u(s) d s: u \in H\right\}\right) .
$$

By Lemma 2.3 for any $t \in[0,+\infty)$, we have

$$
\alpha\left(\left\{\int_{0}^{t} u(s) d s: u \in H\right\}\right) \leq 2 \int_{0}^{t} \alpha(\{u(s): u \in H\}) d s .
$$

Hence, from (2.24) and (2.25), we have

$$
\begin{aligned}
& \alpha\left(\left\{\int_{0}^{+\infty} u(s) d s: u \in H\right\}\right) \\
& \quad=\lim _{t \rightarrow+\infty} \alpha\left(\left\{\int_{0}^{t} u(s) d s: u \in H\right\}\right) \leq 2 \lim _{t \rightarrow+\infty} \int_{0}^{t} \alpha(\{u(s): u \in H\}) d s=2 \int_{0}^{+\infty} \alpha(\{u(s): u \in H\}) d s .
\end{aligned}
$$

Therefore, (2.15) is satisfied. The proof of Lemma 2.3 is completed. 
Zhenbin Liu et al.

Remark 2.4. Lemma 2.3 generalizes Lemma 2.2 from a finite interval to an infinite interval, and it plays an important role in studying the differential equations defined on an infinite interval. It should be emphasized that Lemma 2.3 has no counterpart in the existing literature.

Lemma 2.5 (see [2, Lemma 7]). Let $\left(H_{1}\right)$ be satisfied, and let $V=\left\{u_{m}\right\} \subset D_{e}^{n-1}[J$, E] be a countable bounded set. Then

$$
\alpha_{D}(A V)=\max \left\{\sup _{t \in J}\left[e^{-t} \alpha\left((A V)^{(i)}(t)\right)\right]: i=0,1, \ldots, n-1\right\}
$$

where $\alpha_{D}(\cdot)$ denotes the Kuratowski measure of noncompactness in $D_{e}^{n-1}[J, E],(A V)^{(i)}=\left\{\left(A u_{m}\right)^{(i)}(t)\right.$ : $\left.u_{m} \in V, m=1,2, \ldots\right\}(i=0,1,2, \ldots, n-1)$.

Lemma 2.6. If condition $\left(H_{1}\right)$ is satisfied, then the operator $A$ is continuous and

$$
A\left(D_{e}^{n-1}[J, P]\right) \subset D_{e}^{n-1}[J, P] .
$$

Proof. For any $u \in D_{e}^{n-1}[J, P]$, we have

$$
\begin{aligned}
(A u)(t)= & \frac{1}{\beta-1}\left[\sum_{j=0}^{n-2}\left(\prod_{s=j}^{n-2} \beta_{s}\right) \frac{t^{j}}{j !}+\frac{t^{n-1}}{(n-1) !}\right] \int_{0}^{+\infty} f\left((s), u(s), u^{\prime}(s), \ldots, u^{(n-1)}(s)\right) d s \\
& +\frac{1}{(n-1) !} \int_{0}^{t}(t-s)^{n-1} f\left(s, u(s), u^{\prime}(s), \ldots, u^{(n-1)}(s)\right) d s, \\
(A u)^{(i)}(t)= & \frac{1}{\beta-1}\left[\sum_{j=i}^{n-2}\left(\prod_{s=j}^{n-2} \beta_{s}\right) \frac{t^{j-i}}{(j-i) !}+\frac{t^{n-i-1}}{(n-i-1) !}\right] \int_{0}^{+\infty} f\left(s, u(s), \ldots, u^{(n-1)}(s)\right) d s \\
& +\frac{1}{(n-i-1) !} \int_{0}^{t}(t-s)^{n-i-1} f\left((s), u(s), \ldots, u^{(n-1)}(s)\right) d s, \quad i=1, \ldots, n-1, \\
(A u)^{(n)}(t)= & f\left(t, u(t), u^{\prime}(t), \ldots, u^{(n-1)}(t)\right) .
\end{aligned}
$$

So, by (2.30) and condition $\left(\mathrm{H}_{1}\right)$ for $i=0,1, \ldots, n-1$, we have

$$
\begin{aligned}
e^{-t}\left\|(A u)^{(i)}(t)\right\| & \leq \frac{\beta^{*}+\beta}{\beta-1} e^{-t} \sum_{j=i}^{n-1} \frac{t^{j-i}}{(j-i) !} \int_{0}^{+\infty}\left\|f\left((s), u(s), u^{\prime}(s), \ldots, u^{(n-1)}(s)\right)\right\| d s \\
& \leq \frac{\beta^{*}+\beta}{\beta-1} e^{-t} \sum_{j=i}^{n-1} \frac{t^{j-i}}{(j-i) !}\left(a^{*}+\sum_{i=0}^{n-1} b_{i}^{*}\|u\|_{D}\right),
\end{aligned}
$$

where $a^{*}=\int_{0}^{+\infty} a(t) d t$. Hence, $\lim _{t \rightarrow+\infty} e^{-t}\left\|(A u)^{(i)}(t)\right\|=0$. Therefore, (2.28) is satisfied. 
Now we show that the operator $A$ is continuous. Let $u_{m}, \bar{u} \in D_{e}^{n-1}[J, P],\left\|u_{m}-\bar{u}\right\|_{D} \rightarrow$

0 . Then $r=\sup _{m}\left\|u_{m}\right\|_{D}<+\infty$ and $\|\bar{u}\| \leq r$. By the integrability of $a(t)$ and $e^{t} b_{i}(t)$ $(i=0,1, \ldots, n-1)$ for any $\varepsilon>0$, there exists $T>0$ such that

$$
\frac{\beta^{*}+\beta}{\beta-1}\left[2 \int_{T}^{+\infty} a(t) d t+2 r n \int_{T}^{+\infty} e^{t} b_{i}(t) d t\right]<\frac{\varepsilon}{2} .
$$

On the other hand, by the continuity of $f$, it is easy to see that

$$
\int_{0}^{T}\left\|f\left(s, u_{m}(s), \ldots, u_{m}^{(n-1)}(s)\right)-f\left(s, \bar{u}(s), \ldots, \bar{u}^{(n-1)}(s)\right)\right\| d s \longrightarrow 0, \quad m \longrightarrow+\infty .
$$

Hence, for the above $\varepsilon>0$, there exists a natural number $m_{0}$ such that for any $m>m_{0}$,

$$
\frac{\beta^{*}+\beta}{\beta-1} \int_{0}^{T}\left\|f\left(s, u_{m}(s), \ldots, u_{m}^{(n-1)}(s)\right)-f\left(s, \bar{u}(s), \ldots, \bar{u}^{(n-1)}(s)\right)\right\| d s<\frac{\varepsilon}{2} .
$$

Thus, from (2.13), (2.32), (2.34), and $\left(H_{1}\right)$ for any $m>m_{0}$, we obtain

$$
\begin{aligned}
\left\|A u_{m}-A \bar{u}\right\|_{D} & \leq \frac{\beta^{*}+\beta}{\beta-1} \int_{0}^{+\infty}\left\|f\left(s, u_{m}(s), \ldots, u_{m}^{(n-1)}(s)\right)-f\left(s, \bar{u}(s), \ldots, \bar{u}^{(n-1)}(s)\right)\right\| d s \\
& \leq \frac{\varepsilon}{2}+\frac{\beta^{*}+\beta}{\beta-1}\left[2 \int_{T}^{+\infty} a(t) d t+\sum_{i=0}^{n-1}\left(\left\|u_{m}\right\|_{D}+\|\bar{u}\|_{D}\right) \int_{T}^{+\infty} e^{t} b_{i}(t) d t\right] \\
& \leq \frac{\varepsilon}{2}+\frac{\beta^{*}+\beta}{\beta-1}\left[2 \int_{T}^{+\infty} a(t) d t+2 r n \int_{T}^{+\infty} e^{t} b_{i}(t) d t\right]<\varepsilon .
\end{aligned}
$$

Therefore, the continuity of $A$ is proved. So, the proof of Lemma 2.6 is completed.

Lemma $2.7\left([10,12]\right.$ (Mönch)). Let $V$ be a closed and convex subset of $E$ and $x_{0} \in V$. Assume that operator $A: V \rightarrow V$ has the following property:

$$
C \subset V \text { countable, } \quad C \subset \overline{\mathrm{CO}}\left(\left\{x_{0}\right\} \cup A(V)\right) \Longrightarrow V \text { is relatively compact. }
$$

Then $A$ has a fixed point in $V$.

\section{Main result}

In this section, we present the main results we obtained.

Theorem 3.1. Suppose $\left(H_{1}\right)$ and $\left(H_{2}\right)$ hold. Then BVP (1.1) has at least one nonnegative solution.

Proof. Choose

$$
R>a^{*}\left(\frac{\beta-1}{\beta^{*}+\beta}-\sum_{i=0}^{n-1} b_{i}^{*}\right)^{-1}
$$


and let $V=\left\{u \in D_{e}^{n-1}[J, P]:\|u\|_{D} \leq R\right\}$. Obviously, $V$ is a bounded convex closed set. For any $u \in V$, by (2.28), $\left(\mathrm{H}_{1}\right)$ and the definition of the norm $\|\cdot\|_{e}$, we have

$$
\left\|(A u)^{(i)}\right\|_{e}=\sup _{t \in J}\left\{e^{-t}\left\|(A u)^{(i)}(t)\right\|\right\} \leq \frac{\beta^{*}+\beta}{\beta-1}\left(a^{*}+\sum_{j=0}^{n-1} b_{j}^{*}\|u\|_{D}\right) \leq R, \quad i=0,1, \ldots, n-1,
$$

from which and the definition of the norm $\|\cdot\|_{D}$, we obtain

$$
\|A u\|_{D}=\max \left\{\left\|(A u)^{(i)}\right\|_{e}: 0 \leq i \leq n-1\right\} \leq R .
$$

Therefore, by Lemma 2.6, we conclude that $A$ is a continuous operator from $V$ to $V$.

Next, we prove that $C$ is a relatively compact set if $C \subset V$ is a countable set satisfying $C \subset \overline{\mathrm{co}}(\{u\} \cup A C)$ for some $u \in V$. From this and the properties of the Kuratowski measure of noncompactness in $D_{e}^{n-1}[J, E]$, we have

$$
\alpha_{D}(C) \leq \alpha_{D}(A C)
$$

On the other hand, by (2.28), Lemmas 2.2-2.5, and $\left(H_{2}\right)$ for any $t \in J$ and $0 \leq i \leq n-1$, we have

$$
\begin{aligned}
e^{-t} \alpha\left((A C)^{(i)}(t)\right) & \leq \frac{\beta^{*}+\beta}{\beta-1} 2 \int_{0}^{+\infty} \alpha\left(f\left(s, C(s), C^{\prime}(s), \ldots, C^{(n-1)}(s)\right)\right) d s \\
& \leq \frac{2\left(\beta^{*}+\beta\right)}{\beta-1} \sum_{j=0}^{n-1} l_{j} \alpha_{D}(C) .
\end{aligned}
$$

Thus, by (3.4), (3.5), and Lemma 2.5, we get

$$
\alpha_{D}(C) \leq \alpha_{D}(A C) \leq\left[\frac{2\left(\beta^{*}+\beta\right)}{\beta-1} \sum_{i=0}^{n-1} l_{i}\right] \alpha_{D}(C)
$$

Hence, by $\left(H_{2}\right)$, we have $\alpha_{D}(C)=0$, that is, $C$ is a relatively compact set in $D_{e}^{n-1}[J, E]$. Therefore, by Lemma 2.7, we conclude that $A$ has at least one fixed point in $V \subset D_{e}^{n-1}[J, P]$, that is, $\operatorname{BVP}(1.1)$ has at least one solution in $D_{e}^{n-1}[J, P] \cap C^{n}[J, E]$.

\section{An example}

Example 4.1. Consider the following boundary value problem for a second-order differential equation defined on an infinite interval:

$$
\begin{gathered}
u_{n}^{\prime \prime}(t)=e^{-t}+e^{-14 t} u_{n}(t)+e^{-16 t} u_{n+1}^{\prime}(t), \quad t \in J, \\
u_{n}(0)=u_{n+1}^{\prime}(0) \\
u_{n}^{\prime}(+\infty)=2 u_{n}^{\prime}(0), \quad n=1,2,3, \ldots
\end{gathered}
$$




\section{Conclusion}

BVP (4.1) has at least one nonnegative solution.

Proof. Let

$$
E=l^{1}=\left\{u=\left(u_{1}, u_{2}, \ldots, u_{n}, \ldots\right): \sum_{n=0}^{\infty}\left\|u_{n}\right\|<\infty\right\}
$$

with norm $\|u\|=\sum_{n=0}^{\infty}\left\|u_{n}\right\| . P=\left\{u=\left(u_{1}, u_{2}, \ldots, u_{n}, \ldots\right) \in l^{1}: u_{n} \geq 0, n=1,2, \ldots\right\}$ is a cone in a Banach space $E$ and BVP (4.1) is in the form of BVP (1.1) in $E$ with $n=2$. In this situation, $J=[0, \infty) u=\left(u_{1}, \ldots, u_{n}, \ldots\right), v=\left(v_{1}, \ldots, v_{n}, \ldots\right)$, and $f=\left(f_{1}, \ldots, f_{n}, \ldots\right)$ in which

$$
f_{n}(t, u, v)=e^{-t}+e^{-14 t} u_{n}(t)+e^{-16 t} u_{n+1}^{\prime}(t)
$$

It is clear that $f \in C[J \times E \times E, E]$. For any $t \in J, u, v \in P$, we have

$$
\left|f_{n}(t, u, v)\right| \leq e^{-t}+e^{-14 t} u_{n}(t)+e^{-16 t} u_{n+1}^{\prime}(t) .
$$

So we get

$$
\|f(t, u, v)\| \leq a(t)+b_{0}(t)\|u\|+b_{1}(t)\|v\|,
$$

where $a_{0}(t)=e^{-t}, b_{0}(t)=e^{-14 t}, b_{1}(t)=e^{-16 t}$. From (4.1) and (5.3), we have

$$
\begin{aligned}
& a^{*}=\int_{0}^{+\infty} e^{-t} d t=1, \quad b_{0}^{*}=\int_{0}^{+\infty} e^{t} e^{-14 t} d t=\frac{1}{13} \\
& b_{1}^{*}=\int_{0}^{+\infty} e^{t} e^{-16 t} d t=\frac{1}{15}, \quad \beta^{*}=\beta_{0}=1 .
\end{aligned}
$$

So, we have

$$
\frac{\beta-1}{\beta^{*}+\beta}=\frac{2-1}{1+2}=\frac{1}{3}>\frac{1}{13}+\frac{1}{15}=b_{0}^{*}+b_{1}^{*} .
$$

Hence, the condition ( $\left.\mathrm{H}_{1}\right)$ is satisfied. By (5.3) for any bounded sets $D_{1}, D_{2} \subset l^{1}$, we get

$$
\alpha\left(f\left(t, D_{1}, D_{2}\right)\right) \leq l_{1}(t) \alpha\left(D_{1}\right)+l_{2}(t) \alpha\left(D_{2}\right),
$$

where $l_{1}(t)=e^{-14 t}, l_{2}(t)=e^{-16 t}$. So

$$
\begin{gathered}
l_{1}=\int_{0}^{+\infty} e^{t} l_{1}(t) d t=\frac{1}{13}<+\infty, \quad l_{2}=\int_{0}^{+\infty} e^{t} l_{2}(t) d t=\frac{1}{15}<+\infty, \\
\frac{2\left(\beta^{*}+\beta\right)}{\beta-1}\left(l_{1}+l_{2}\right)=\left(\frac{1}{13}+\frac{1}{15}\right) \times 6<1 .
\end{gathered}
$$

Hence, the condition $\left(\mathrm{H}_{2}\right)$ is satisfied. Therefore, our conclusion follows from Theorem 3.1. 


\section{Acknowledgments}

The authors are grateful to the referees whose comments have led to a number of significant improvements of the paper. The first and second authors are supported financially by the National Natural Science Foundation of China (10771117) and the State Education Commission Doctoral Foundation of China (20060446001). The third author is supported financially by the Australia Research Council through an ARC Discovery Project Grant.

\section{References}

[1] D. Guo, "Second order integro-differential equations of Volterra type on unbounded domains in Banach spaces," Nonlinear Analysis: Theory, Methods E Applications, vol. 41, no. 3-4, pp. 465-476, 2000.

[2] D. Guo, "Existence of solutions for $n^{\text {th }}$ order impulsive integro-differential equations in a Banach space," Nonlinear Analysis: Theory, Methods E Applications, vol. 47, no. 2, pp. 741-752, 2001.

[3] D. Guo, "Multiple positive solutions for first order nonlinear integro-differential equations in Banach spaces," Nonlinear Analysis: Theory, Methods E Applications, vol. 53, no. 2, pp. 183-195, 2003.

[4] D. Guo, "Multiple positive solutions for $n^{\text {th }}$-order impulsive integro-differential equations in Banach spaces," Nonlinear Analysis: Theory, Methods E Applications, vol. 60, no. 5, pp. 955-976, 2005.

[5] W. Guo, "A generalization of the Ascoli-Arzela theorem and its application," Journal of Systems Science and Mathematical Sciences, vol. 22, no. 1, pp. 115-122, 2002 (Chinese).

[6] Y. Liu, "Boundary value problems for second order differential equations on unbounded domains in a Banach space," Applied Mathematics and Computation, vol. 135, no. 2-3, pp. 569-583, 2003.

[7] X. Q. Zhang, "Infinite boundary value problems for first-order differential equations in a Banach space," Mathematica Applicata, vol. 18, no. 1, pp. 153-160, 2005 (Chinese).

[8] Y. Zhou, "The solution of extremal value problem for first order differential equation," Journal of Systems Science and Mathematical Sciences, vol. 19, no. 3, pp. 264-267, 1999 (Chinese).

[9] K. Deimling, Nonlinear Functional Analysis, Springer, Berlin, Germany, 1985.

[10] D. Guo, V. Lakshmikantham, and X. Liu, Nonlinear Integral Equations in Abstract Spaces, vol. 373 of Mathematics and Its Applications, Kluwer Academic, Dordrecht, The Netherlands, 1996.

[11] H.-P. Heinz, "On the behaviour of measures of noncompactness with respect to differentiation and integration of vector-valued functions," Nonlinear Analysis: Theory, Methods \& Applications, vol. 7, no. 12, pp. 1351-1371, 1983.

[12] H. Mönch, "Boundary value problems for nonlinear ordinary differential equations of second order in Banach spaces," Nonlinear Analysis: Theory, Methods E Applications, vol. 4, no. 5, pp. 985-999, 1980.

[13] H. Mönch and G.-F. von Harten, "On the Cauchy problem for ordinary differential equations in Banach spaces," Archiv der Mathematik, vol. 39, no. 2, pp. 153-160, 1982. 


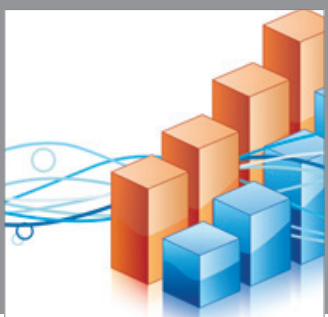

Advances in

Operations Research

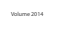

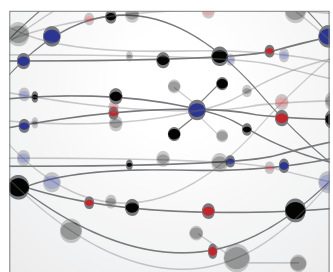

\section{The Scientific} World Journal
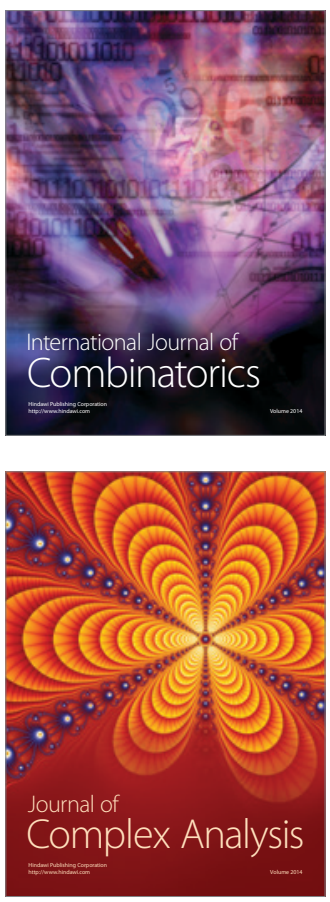

International Journal of

Mathematics and

Mathematical

Sciences
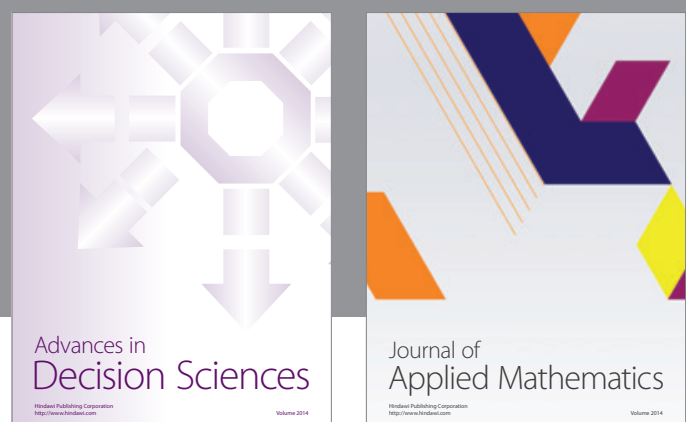

Journal of

Applied Mathematics
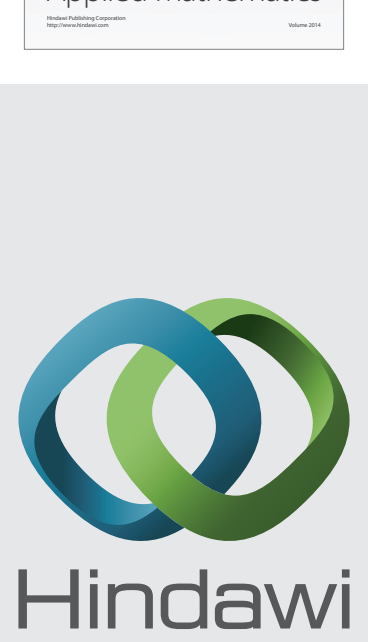

Submit your manuscripts at http://www.hindawi.com
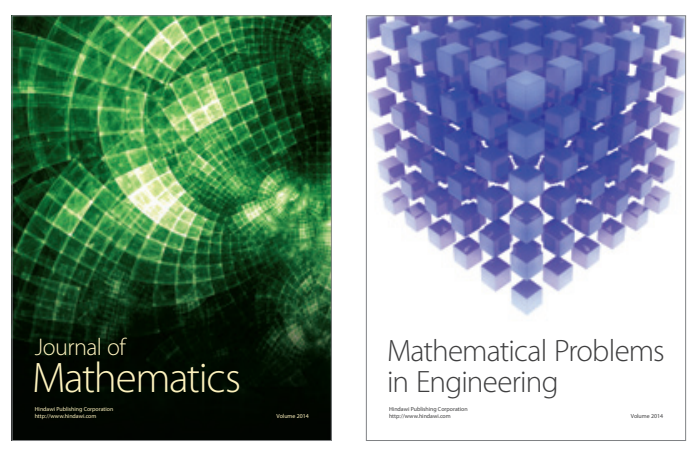

Mathematical Problems in Engineering
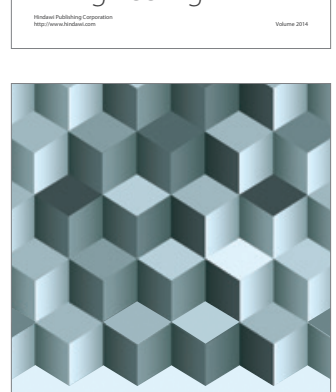

Journal of

Function Spaces
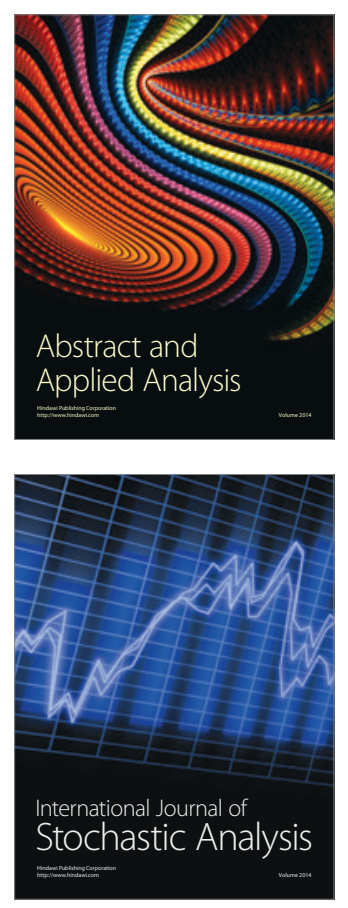

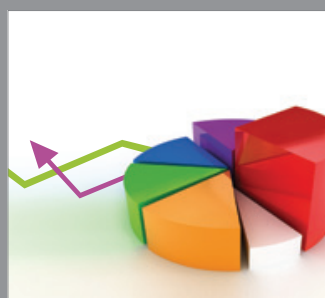

ournal of

Probability and Statistics

Promensencen
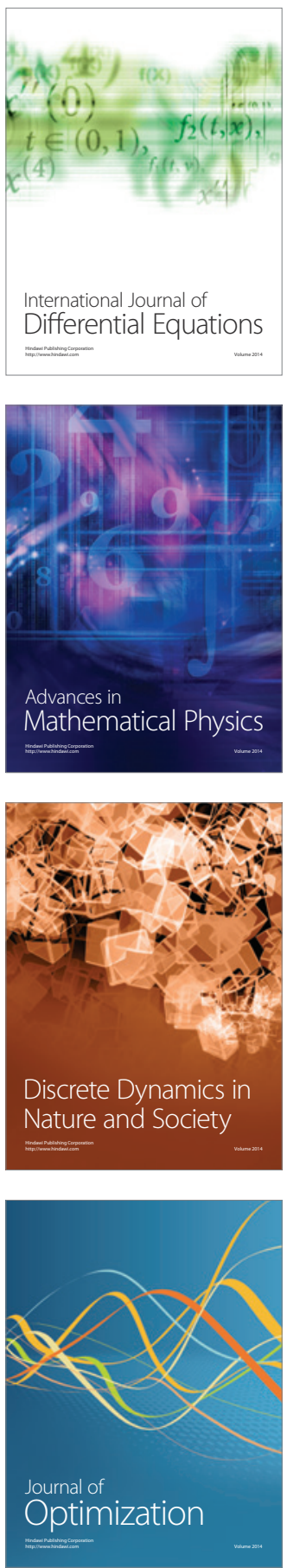